\title{
Análisis de la creatividad y la flexibilidad cognitiva en adolescentes en un espacio de innovación educativa
}

Analysis of creativity and cognitive flexibility in adolescents in a space of educational innovation

\author{
Franco Donadel* \\ Gabriela Morelato** \\ Celina Korzeniowski***
}

\section{Resumen}

El estudio exploró la capacidad creativa - fluidez y flexibilidad creativa - y la flexibilidad cognitiva en un grupo de adolescentes que participaron regularmente en las actividades de un centro de innovación educativa, analizando su posible relación. El diseño implementado fue no experimental de tipo transversal cuantitativo de alcance exploratorio-descriptivo. La muestra se compuso por 19 adolescentes de entre 11 y 16 años que asistieron con regularidad a un centro de innovación educativa. Los instrumentos fueron el Test CREA de Inteligencia Creativa (Corbalán et al., 2003) y el Subtest de Formación de Conceptos de la Batería III Cog Woodcock-Muñoz (Woodcock et al., 2005). Los resultados señalaron en el grupo analizado un alto nivel de creatividad en comparación con el baremo argentino. A su vez se hallaron asociaciones entre las variables fluidez creativa $y$ flexibilidad creativa. Flexibilidad cognitiva se asoció con flexibilidad creativa, aunque no registró asociación con fluidez creativa. Los hallazgos, ligados al tipo de metodología implementada en el centro educativo, abren nuevos interrogantes e hipótesis sobre las prácticas pedagógicas y las características de los adolescentes que asisten a estos espacios. Palabras clave: Creatividad, Flexibilidad cognitiva ; Innovación educativa.

\section{Abstract}

The study explored the creative capacity -creative fluency and flexibility - and cognitive flexibility in a group of adolescents who regularly participated in the activities of an educational innovation center, analyzing their possible relationship. The implemented design was non-experimental of a quantitative cross-sectional type of exploratory-descriptive scope. The sample consisted of 19 adolescents between the

*Lic. en Psicología. Universidad del Aconcagua. Facultad de Psicología, Mendoza, Argentina

** Dra. en Psicología. Universidad del Aconcagua. Facultad de Psicología, Mendoza, Argentina; Consejo Nacional de Investigaciones Científicas y Técnicas (CONICET). Instituto de Ciencias Humanas Sociales y Ambientales (INCIHUSA),

*** Dra. en Psicología. Universidad del Aconcagua. Facultad de Psicología, Mendoza, Argentina; Consejo Nacional de Investigaciones Científicas y Técnicas (CONICET). Instituto de Ciencias Humanas Sociales y Ambientales (INCIHUSA)

Doi: https://doi.org/10.46553/RPSI.17.34.2021.p7-20

Fecha de Recepción: 22 de septiembre de 2020 Fecha de Aceptación: 13 de julio de 2021 
ages of 11 and 16 who regularly attended an educational innovation center. The instruments were the CREA Test of Creative Intelligence (Corbalán et al., 2003) and the Batería III Cog Woodcock-Muñoz Concept Formation Subtest (Woodcock et al., 2005). The results indicated a high level of creativity in the analyzed group compared to the Argentine scale. In turn, associations were found between the variables creative fluency and creative flexibility. Cognitive flexibility was associated with creative flexibility, although it did not register an association with creative fluency. The findings, linked to the type of methodology implemented in the educational center, open new questions and hypotheses about the pedagogical practices and the characteristics of the adolescents who attend these spaces.

Key words: Creativity, Cognitive flexibility, Educational innovation.

\section{Introducción}

El presente estudio surgió a partir de una experiencia ligada al trabajo con niños, niñas y adolescentes (NNyA) donde se tuvo la oportunidad de ejercer el rol de docentefacilitador en un contexto de educación no formal. Este rol consistió en llevar a cabo tareas enfocadas al acompañamiento y guía de los NNyA de modo personalizado acorde a sus motivaciones particulares y sus diversas formas de transitar la propuesta pedagógica. El espacio donde se desarrollaron fue un centro de innovación educativa y tecnológica, sostenido de forma compartida entre el Estado Nacional y Provincial (MendozaArgentina) que tuvo como objetivo fomentar el desarrollo de habilidades y competencias en NNyA vinculadas con la adaptabilidad, el pensamiento crítico, el trabajo en equipo y la creatividad mediante talleres y proyectos. Al reflexionar en torno a las prácticas de la educación es frecuente encontrar menciones y desarrollos acerca de la innovación educativa. La ONU (2016) declaró que la innovación constituye un cambio que incide en algún aspecto estructural de la educación para mejorar su calidad, lo cual puede ocurrir a nivel de dispositivos espaciales como las aulas, de las distintas instituciones educativas y del sistema escolar. Asimismo, es importante considerar que no cualquier cambio o modificación implica innovar, pues la innovación debe ser intencional, planificada y evaluada (Zavala-Guirado et al. 2019). Asimismo, aunque los proyectos y acciones de innovación educativa son susceptibles de aplicar a cualquier contexto educativo, no obstante, en entornos de educación no formal se suelen generar terrenos más fértiles para la innovación.

En Argentina la educación no formal está contemplada por la Ley de Educación Nacional 26.206. La misma pretende transmitir aprendizajes que la formal no completa o, alternativamente, abrir otros caminos para el aprovechamiento de las capacidades y recursos de la comunidad en los planos de la cultura, el arte, el deporte, la investigación científica y tecnológica.

En el ámbito local se desarrollan y llevan a cabo proyectos de innovación educativa desde un modelo pedagógico que sienta sus bases en el enfoque constructivista. Este considera la autogestión del aprendizaje y la construcción del conocimiento a partir de experiencias previas (Saldarriaga-Zambrano et al., 2016). Tal perspectiva posibilita la implementación de otros modelos 
pedagógicos tales como el conectivismo, el aprendizaje basado en proyectos y la cultura maker (Siemens, 2004; Blank, 1997; Harwell, 1997; Martí et al., 2010).

En general el objetivo de estos planes es el desarrollo de habilidades blandas que se consideran importantes para el futuro. Tales habilidades constituyen capacidades que se encuentran en estrecha relación con las habilidades cognitivas dado que se retroalimentan de modo permanente (Matus y Gutiérrez, 2012).

Una de las habilidades cognitivas relevantes es la flexibilidad cognitiva. La misma permite abordar un problema desde diferentes perspectivas y ajustar los cambios rápidamente a las demandas o prioridades empleando estrategias alternativas (Diamond, 2014; Hoyos De Los Ríos et al., 2013). Además, es considerada una capacidad para cambiar intermitentemente el foco de atención sobre una o varias reglas, interactuando necesariamente con los procesos de inhibición y con la memoria de trabajo (Slachevsky et al., 2005).

Por otra parte, se hace evidente que estos espacios intentan particularmente fomentar el proceso creativo. Cuando nos referimos a la creatividad se parte de lo postulado por Guilford (1950) quien considera a la conducta creativa como la que integra actividades tales como invención, elaboración, organización, composición y planificación. Se la considera como una actividad intelectual que forma parte del pensamiento o producción divergente; es decir, de la capacidad que tiene una persona de pensar en varias y diferentes alternativas de solución a un problema determinado.

La capacidad creativa puede ser desarrollada en la mayoría de las personas donde el medio social participa inhibiendo o favoreciendo a la misma (Morelato et al., 2014). Duarte Briceño (2003), agrega que una atmósfera creativa es aquella que propicia un estado óptimo de activación en los procesos de pensamiento, y al mismo tiempo, incrementa la producción grupal. En esta línea los indicadores de creatividad más utilizados son fluidez creativa, flexibilidad mental o creativa y originalidad (Corbalán Berná, 2008; Morelato et al., 2014).

Investigaciones realizadas en otros países de la región dan cuenta de la necesidad de incluir en los dispositivos de educación elementos que fomenten el desarrollo de la creatividad. Los autores señalan que el uso de pedagogías que se caractericen por ser dinámicas, activas, implicativas y motivadoras podrían ser favorables para que los estudiantes se encuentren más capacitados frente a los desafíos que implican la educación superior y el trabajo productivo. Asimismo, los estudios destacan que en las instituciones donde las orientaciones del modelo pedagógico se aproximaban hacia las orientaciones metodológicas para el fomento de creatividad, los docentes configuraban sus prácticas de enseñanza de forma consciente y planificada orientándose hacia el fomento de la capacidad creativa en un alto grado (Muñoz, 2011; Klimenko et al., 2015).

En ese marco y con base en la experiencia ligada al trabajo en espacios de innovación, se pudo observar que los adolescentes que asistían con regularidad a este espacio presentaban particularidades tales como interés por lo nuevo, motivación por crear y deseo de interactuar sin importar la edad de sus compañeros. Además, se observaba plasticidad y flexibilidad en las ideas y propósitos, lo cual resultaba 
interesante, considerando que se trataba de grupos heterogéneos. Esto llevó a la pregunta acerca de si estos niños, niñas y adolescentes manifestaban preponderantemente un buen nivel de creatividad y/o contaban con características cognitivas que los hacían especialmente flexibles.

Por otro lado, no se hallaron suficientes datos que pudieran describir fenómenos y experiencias ocurridas en ámbitos educativos ajenos y marcadamente distintos a la educación formal, ya que son escasas las investigaciones en innovación educativa realizadas en el ámbito regional y nacional. Así, surgió la idea de estudiar la relación entre dos de los aspectos que resultaban relevantes en la observación empírica: la creatividad y la flexibilidad cognitiva. De este modo, aunque la naturaleza del espacio hacía factible explorar sólo algunos factores debido a lo novedoso del mismo, se estimó que la posibilidad de explorarlos resultaba importante a modo preliminar y a fin de abrir la posibilidad, a posteriori, de profundizar los resultados.

Por lo tanto, el objetivo de este estudio fue evaluar la flexibilidad cognitiva y la capacidad creativa en los adolescentes que participan regularmente en las actividades de centro de innovación educativa, analizando su posible relación.

\section{Diseño de investigación y método}

La investigación se llevó a cabo desde un diseño no experimental de tipo transversal de enfoque cuantitativo (Hernández Sampieri et al., 2014; León y Montero, 2015). El alcance fue definido como exploratorio debido a la poca investigación sistematizada efectuada en ámbitos locales — provincial y nacionalpor lo que marca la necesidad de realizar un estudio que sirva para sentar las bases para posteriores investigaciones más específicas.

\section{Participantes}

En la presente investigación participaron 19 adolescentes (68,4\% mujeres y $31,6 \%$ varones) con edades de entre 11 y 16 años $(\mathrm{M}=12,16 ; \mathrm{DS}=1,803)$. Fueron considerados adolescentes según los términos de la OMS (2019) quien define a la adolescencia como un período de crecimiento y desarrollo humano que se produce después de la niñez y antes de la edad adulta, entre los 10 a 19 años. Vale destacar que todos pertenecían a grupos naturales - es decir, previamente conformados- y que tenían en común coincidir semanalmente en las actividades propuestas por el espacio de innovación educativa. Estas actividades eran elegidas por motivación propia en el marco del dispositivo de educación orientado a la planificación de proyectos, talleres y actividades que comprendían a adolescentes de esas edades. Esta amplitud tenía el fin de propiciar relaciones donde prime la diversidad — de edades, géneros, intereses, etc.

Todos pertenecían a contextos socioeconómicos y educativos de nivel medio, de la zona urbana de gran Mendoza. Fueron seleccionados de manera intencional, no probabilística, siguiendo el criterio por el cual asistieran al centro educativo con una frecuencia mínima de dos visitas semanales por un tiempo no menor a tres meses. Se excluyeron participantes que concurrieran al centro con mayor antigüedad, pero con menor frecuencia o una frecuencia no regular.

El lugar donde se llevó a cabo la actividad fue el centro de innovación 
educativa. El espacio estaba ubicado en la zona centro de la provincia de Mendoza, Argentina y contó con características particulares, tales como un régimen de asistencia voluntaria - no obligatoria - con participantes con edades entre 6 y 18 años. Se trataba de un espacio abierto, donde había laboratorios equipados con tecnología de última generación que podían utilizarse libremente, según consignas e intereses de los participantes con ayuda y acompañamiento de los facilitadores. Se proponía a los participantes planificar, realizar, evaluar y mostrar proyectos propios.

\section{Instrumentos}

\section{Test CREA Inteligencia Creativa (Corbalán et al., 2003)}

Utilizado para evaluar la capacidad creativa o creatividad; el objetivo de este es medir la inteligencia creativa, evaluando la capacidad del sujeto para elaborar preguntas a partir de un material gráfico suministrado. La técnica ofrece una medida indirecta de la creatividad, en tanto que fuerza a activar los mecanismos que participan en el acto creativo. Consta de tres láminas $(\mathrm{A}, \mathrm{B}$ y $\mathrm{C})$ a partir de las cuales el sujeto debe generar todo tipo de preguntas que le sugieran los dibujos que las constituyen. Cada interrogante expresado supone un nuevo esquema cognitivo nacido de la interacción del estímulo con la capacidad de la persona de abrir esa nueva información a toda la que ya dispone. El instrumento centra su interés en la medida fluidez creativa o productividad; siendo ésta la capacidad de dar respuestas a los estímulos presentados. Operacionalmente, remite a la cantidad de ideas generadas por una persona frente a éstos.
Se puntúa concediendo un punto a cada figura, excepto las repetidas o las que no tienen significado alguno. Cuenta con baremación en la población argentina (Corbalán et al., 2003) realizada en un contexto que presenta características sociodemográficas similares a las del grupo estudiado (nivel socioeconómico-educativo medio, de entornos urbanos, de la zona de Córdoba y alrededores).

La flexibilidad creativa fue evaluada siguiendo una modalidad fundamentada en estudios previos (Morelato et al., 2019). Se construyó una serie de diez categorías de respuestas posibles, otorgando un punto por cada categoría utilizada: de situación, de razón, de lugar, calificativas, temporales, de funcionamiento, de señalamiento específico, de material, de cantidad y de alusión a un sujeto. Se clasificaron las respuestas con base en la frecuencia de aparición de las diversas preguntas realizadas por los y las adolescentes del grupo. Posteriormente y a modo de triangulación metodológica, dos jueces, estudiantes avanzados de la carrera de psicología, analizaron las categorías construidas a fin de obtener un criterio de acuerdo respecto de la validez de contenido de estas. Para ello se calculó el índice de validez de contenido (Polit et al., 2007), obteniendo un puntaje de 0.95 , lo cual es considerado adecuado.

\section{Subtest de Formación de Conceptos de la Batería III Cog Woodcock-Muñoz}

Desarrollado por Muñoz Sandoval et al. (2005), es una tarea de aprendizaje controlado y razonamiento categorial basado en los principios de la lógica inductiva, que consiste en presentarle al sujeto una serie de estímulos de la cual debe inferir la regla para 
cada ítem. Es de administración individual y se toma desde los 5 años hasta la adultez. Esta tarea mide la capacidad de razonar de manera abstracta, pensar con flexibilidad, formar conceptos, hacer inferencias, comprender las implicaciones y resolver problemas utilizando información novedosa. En el presente estudio de investigación, este instrumento se utilizó para evaluar la flexibilidad cognitiva. La mediana de la confiabilidad test-retest de este instrumento es rho $=.94$ (Woodcock et al., 2005). La tarea propuesta consiste en la resolución de una serie de rompecabezas gráficos compuestos por una hilera de círculos o cuadrados, los cuales varían en color, tamaño y cantidad. En los rompecabezas algunas figuras están encerradas y otras no. La persona debe identificar la regla que explica por qué los estímulos encerrados son diferentes de los no encerrados. Esta regla va incrementándose en dificultad a medida que se progresa en la tarea. La persona no sólo debe identificar la regla adecuada para cada problema, sino que debe utilizar su flexibilidad cognitiva para cambiar de regla cuando la tarea así lo requiera.

\section{Procedimiento}

Para llevar adelante el estudio se gestionaron las autorizaciones institucionales y se obtuvieron los consentimientos informados de los padres, madres o cuidadores según los criterios éticos y legales. Asimismo, a los y las adolescentes se les propuso dar su asentimiento para el desarrollo de la investigación, independientemente de la firma del consentimiento informado por parte de los cuidadores principales. Posteriormente, se dio lugar a la administración de las técnicas en forma individual en el orden consignado anteriormente, con un descanso de cinco minutos entre técnicas. La administración de ambos instrumentos llevó aproximadamente $25-30$ minutos por participante.

Se analizaron los datos por medio del programa SPSS 22.0 mediante estadísticos descriptivos, intervalos de nivel, prueba de medias y análisis bivariado.

\section{Presentación de resultados}

En relación al análisis descriptivo de la fluidez creativa, se obtuvo una M (media) $=18,47$ y una DS (desviación estándar) = 8,70 . Es destacable que el promedio arrojado para la muestra investigada se ubicó en el percentil 80 del baremo, es decir en un rango alto (Corbalán et al., 2003). A continuación, se realizó una prueba de diferencia entre medias, comparando los resultados obtenidos con el baremo para la población de adolescentes argentinos. Tal comparación (ver tabla 1) arrojó resultados significativos con un tamaño del efecto alto, en coherencia con las observaciones realizadas.

Posteriormente se agruparon los resultados obtenidos por el grupo analizado en el presente estudio según intervalos de nivel acorde a un criterio intramuestral.

En la figura 1 se observa el desempeño de adolescentes en la fluidez o productividad creativa agrupada por rangos y evaluada por el Test CREA. Puede observarse que el mayor porcentaje se concentra en el rango medio $(47,4 \%)$ y alto $(32,6 \%)$.

En la figura 2 se puede observar la flexibilidad creativa en el grupo analizado. Se pone de manifiesto que el $21,1 \%$ (f:4) presenta un nivel de flexibilidad creativa baja; el 36,8\% (f:7); flexibilidad creativa media y el $42,1 \%$ (f: 8 ) flexibilidad creativa 


\section{Tabla 1}

Comparación entre medias de la muestra investigada y el baremo del Test CREA para adolescentes argentinos en fluidez creativa.

\begin{tabular}{lcccccc}
\hline Muestra & Media & N & DS & t & P & d \\
\hline $\begin{array}{l}\text { Adolescentes espacio de } \\
\text { innovación educativa }\end{array}$ & 18,47 & 19 & 8,70 & & & \\
Baremo adolescentes argentinos & 12,86 & 470 & 5,80 & & & \\
\hline
\end{tabular}

\section{Figura 1}

Desempeño de los adolescentes evaluados en las variables fluidez creativa. Distribución por porcentaje

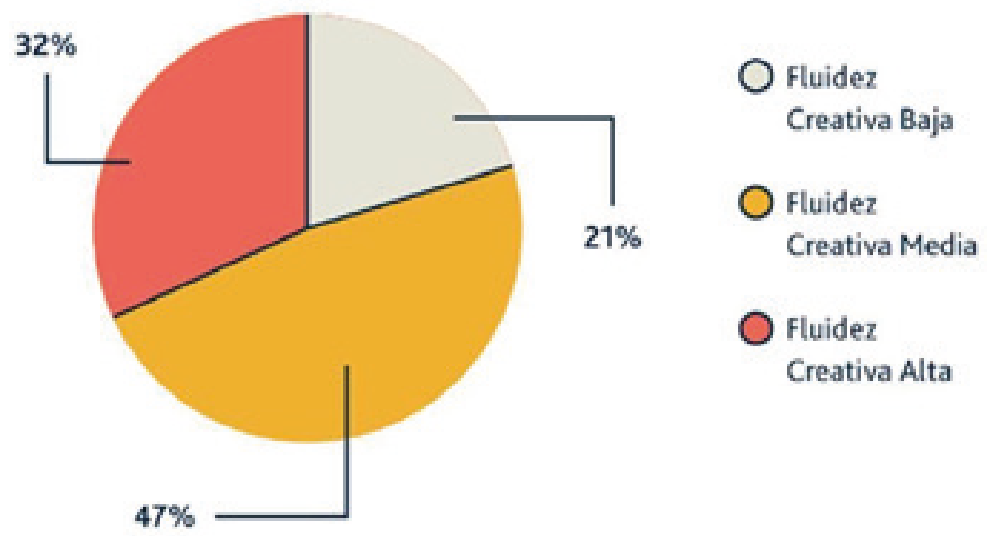

alta para una muestra compuesta por el total de 19 adolescentes.

En relación a la evaluación de la flexibilidad cognitiva, se obtuvo una media de 24,74 (DS = 8,191). Posteriormente a partir de un criterio intramuestral se realizaron intervalos de rango con base en las medidas de posición (cuartiles).

En la figura 3 se puede observar que los porcentajes en flexibilidad cognitiva se hallan equiparados. De este modo el 31,6\%(f:6) obtuvo un nivel bajo en flexibilidad cognitiva; el 36,8\% (f: 7) un nivel medio y el 31,6\%; (f:6) un nivel alto. En otras palabras, los adolescentes que presentaron un puntaje entre 1 y 20 tuvieron un nivel de flexibilidad cognitiva bajo y aquellos que presentaron puntajes comprendidos entre 28 y 38 tuvieron un nivel de flexibilidad cognitiva alto. 
14 Análisis de la creatividad y la flexibilidad cognitiva en adolescentes en un espacio...

\section{Figura 2}

Desempeño de los adolescentes evaluados en la variable flexibilidad creativa. Distribución por porcentaje

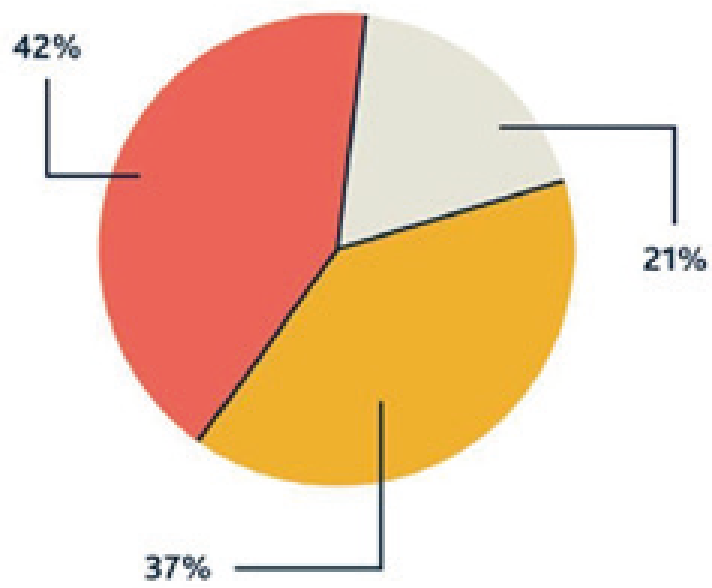

Flexibilidad

Creativa Baja

$\bigcirc$ Flexibilidad

Creativa Media

$\bigcirc$ Flexibilidad

Creativa Alta

\section{Figura 3}

Desempeño de los adolescentes evaluados en la variable flexibilidad cognitiva. Distribución por porcentaje

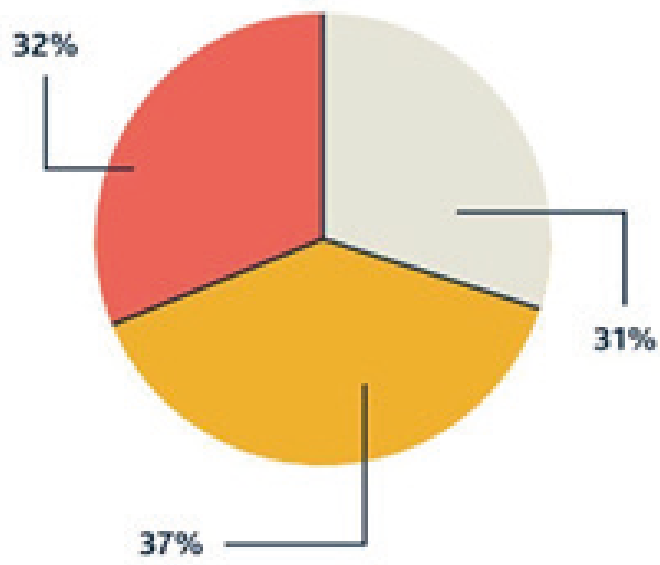

Flexibilidad

Cognitiva Baja

Flexibilidad

Cognitiva Media

Flexibilidad

Cognitiva Alta 
Análisis bivariado

Fluidez creativa y flexibilidad creativa. Para este análisis se halló una asociación significativa $\left(\mathrm{X}^{2}=13,157 ; \mathrm{p}<\right.$ .011; Tau $b=0,670)$ como se detalla en la tabla 2 .

Esta asociación indica que un aumento o disminución de uno de los indicadores remite a un aumento o disminución en el otro, sin que se trate de una relación causa-efecto. Puede observarse que entre fluidez baja y flexibilidad alta $(0 \%)$ no existe frecuencia de porcentaje, es decir, que ningún adolescente con alta flexibilidad tuvo baja productividad. Además, el 62,5\% de los casos de adolescentes con fluidez alta también muestran flexibilidad alta y el $75 \%$ de los casos de fluidez baja también presentan flexibilidad baja.

Flexibilidad creativa y flexibilidad cognitiva. En este análisis se halló una asociación que resultó significativa entre ambas variables $\left(X^{2}=10,445 ; p<.034\right.$; Tau $b$ $=0,170)$. En este sentido, puede observarse como porcentaje más destacable que el $83.3 \%$ de los casos de adolescentes con flexibilidad creativa alta también muestran flexibilidad cognitiva alta. Los resultados se muestran en la tabla 3.

\section{Tabla 2}

Prueba de $X^{2}$ (chí cuadrado) entre Fluidez Creativa y Flexibilidad Creativa en el grupo de adolescentes evaluados

\begin{tabular}{lllll}
\hline Fluidez creativa & $\begin{array}{l}\text { Flex. creativa } \\
\text { baja }\end{array}$ & $\begin{array}{l}\text { Flex. creativa } \\
\text { media }\end{array}$ & $\begin{array}{l}\text { Flex. creativa } \\
\text { alta }\end{array}$ & Recuento \\
\hline Fluidez creativa baja & $\mathbf{7 5 , 0 \% ( 3 )}$ & $14,3 \%(1)$ & $0 \%(0)$ & $21,1 \%(4)$ \\
Fluidez creativa media & $25,0 \%(1)$ & $\mathbf{7 1 , 4 \% ( 5 )}$ & $37,5 \%(3)$ & $47,4 \%(9)$ \\
Fluidez creativa alta & $0 \%(0)$ & $14,3 \%(1)$ & $\mathbf{6 2 , 5 \% ( 5 )}$ & $31,6 \%(6)$ \\
Recuento & $100 \%(4)$ & $100 \%(7)$ & $100 \%(8)$ & $100 \%(19)$ \\
\hline
\end{tabular}

Nota. $\left(\chi^{2}=13,157 ; \mathrm{p}<.011 ;\right.$ Tau $\left.b=0,670\right)$

\section{Tabla 3}

Prueba de $X^{2}$ (chi cuadrado) entre Flexibilidad Creativa y Flexibilidad Cognitiva en el grupo de adolescentes evaluados

\begin{tabular}{lllll}
\hline Flexibilidad creativa & $\begin{array}{l}\text { Flex. cognitiva } \\
\text { baja }\end{array}$ & $\begin{array}{l}\text { Flex. cognitiva } \\
\text { media }\end{array}$ & $\begin{array}{l}\text { Flex. cognitiva } \\
\text { alta }\end{array}$ & Recuento \\
\hline $\begin{array}{l}\text { Flexibilidad creativa baja } \\
\text { Flexibilidad creativa }\end{array}$ & $66 \%(0)$ & $42,9 \%(3)$ & $16,7 \%(1)$ & $21,1 \%(4)$ \\
media & $42,9 \%(3)$ & $0 \%(0)$ & $47,4 \%(7)$ \\
$\begin{array}{l}\text { Flexibilidad creativa alta } \\
\text { Recuento }\end{array}$ & $33,3 \%(2)$ & $14,3 \%(1)$ & $\mathbf{8 3 , 3 \% ( 5 )}$ & $31,6 \%(8)$ \\
\hline
\end{tabular}

Nota. $\left(\chi^{2}=10,445 ; \mathrm{p}<.034 ;\right.$ Tau $\left.b=0,170\right)$ 
Análisis de la creatividad y la flexibilidad cognitiva en adolescentes en un espacio...

Por otra parte, entre las variables flexibilidad cognitiva y fluidez creativa no se halló relación significativa $\left(\mathrm{X}^{2}=6,798 ; \mathrm{p}<\right.$ .147 ; Tau $b=0,703)$.

\section{Discusión}

La fluidez creativa, como indicador de creatividad —a la par de la flexibilidad creativa-, arroja resultados relevantes a la hora de considerar los objetivos planteados. En los puntajes directos de fluidez creativa, la media $(\mathrm{M}=18,47 ; \mathrm{DS}=8,707)$ se ubica, según los baremos para adolescentes argentinos, dentro del rango de fluidez creativa alta, más específicamente en el percentil 80 . Lo hallado podría relacionarse con el ambiente de aprendizaje, que tiene las condiciones sugeridas por López Martínez (2008) para favorecer al pensamiento creativo. Tales condiciones forman parte de las bases conceptuales en las que se apoya la institución donde se realiza la experiencia, las cuales están vinculadas al fomento de la exploración y el autoconocimiento, a la educación en torno a la tolerancia a los conflictos y frustraciones, a un modelo educativo que permita la libertad de elección y la autonomía, a la estimulación de la actitud lúdica, de la espontaneidad creativa, a la ejercitación de la creatividad social a través de la cual se revelan nuevas relaciones y a la realización de actividades en las que se provoque la fantasía. Por lo expuesto, estos resultados pueden ser valorados para futuras investigaciones ya que es posible pensar que el desarrollo de estas capacidades guarde alguna relación con el tránsito por la experiencia educativa de las características mencionadas. Asimismo, podría también considerarse que adolescentes con mayor creatividad buscan asistir a espacios de este estilo para así potenciar sus capacidades.

En la misma línea es posible tomar las consideraciones de Vygotsky (citado en Limiñana Gras, 2008) donde plantea la necesidad de ampliar la experiencia del niño o niña ya que mientras más vea, escuche y viva, mayor será su actividad creativa. Es importante considerar que los aspectos contribuyentes al desarrollo de la inteligencia creativa que se detallan con anterioridad se constituyen como aspectos valorados por parte de los asistentes y en muchos casos forman parte de la motivación para la asistencia (Donadel, 2019).

Por otra parte, la flexibilidad creativa presenta el mayor porcentaje $(42,11 \%)$ en el rango de nivel alto. Tal resultado va en la línea de lo detallado con anterioridad y podría ligarse a la modalidad y al funcionamiento de la institución educativa. No obstante, como se sugirió anteriormente, también surge la pregunta a modo de hipótesis acerca de las características de personalidad de los adolescentes que eligen estos espacios y los transitan con frecuencia, lo cual debería estudiarse en trabajos posteriores.

En el análisis bivariado, se halló una asociación significativa entre la fluidez creativa y la flexibilidad creativa. Tal resultado implica en primer lugar la existencia de una coherencia interna entre el puntaje obtenido por el instrumento, aplicado según lo señalan los autores, y el puntaje de flexibilidad que fue construido especialmente con base en estudios previos (Morelato et al., 2014; Morelato et al., 2013). En este sentido, dota de mayor validez a la categorización construida a partir de la técnica administrada. Por otro lado, permite también considerar que, a mayor índice de fluidez creativa, se 
incrementa el índice de flexibilidad creativa en el grupo de adolescentes evaluado.

La medida de flexibilidad cognitiva, obtenida a través del Sub Test III de Formación de Conceptos de la Batería Woodcock-Muñoz, arrojó para la muestra una media de 24,74 (DS=8,191). La técnica empleada, al no contar con baremación para la población argentina, no permite una interpretación comparativa de los resultados de la muestra con los de la población. No obstante, se realizó una distribución intramuestral por intervalos de nivel bajo-medio-alto, dando por resultado una distribución equitativa entre los tres niveles, conservando un porcentaje más elevado $(36,84 \%)$ en el nivel medio.

En esa línea de análisis, se halló una relación entre las medidas de flexibilidad cognitiva y la flexibilidad creativa. Estos hallazgos son consistentes con lo expuesto hasta ahora y podrían indicar la importancia de generar un ambiente donde se fomente la capacidad de pensar muchas alternativas frente a los problemas.

Para ampliar lo expuesto, se destaca la necesidad de analizar en posteriores estudios la implicancia del modelo pedagógico que considera al aprendizaje basado en proyectos con aspectos importantes del desarrollo, tales como la creatividad y la flexibilidad cognitiva. La flexibilidad cognitiva, como una de las funciones ejecutivas de relevancia, cobra importancia en la adolescencia debido a que favorece el desarrollo del funcionamiento ejecutivo, el desarrollo creativo y una mejora de la habilidad para la resolución de problemas interpersonales (Maddio y Greco, 2010). Desde este punto de vista, es posible considerar lo argumentado por
Korzeniowski (2011) al manifestar que las experiencias ofrecidas por la institución educativa resultan de amplia riqueza para el desarrollo del funcionamiento ejecutivo, ya que se desarrollan en períodos sensibles para la promoción y estimulación. Por otra parte, no se hallaron asociaciones entre los índices de flexibilidad cognitiva y la fluidez creativa. El resultado podría vincularse a que el grupo analizado es muy pequeño por lo que sería conveniente replicar el estudio a mayor escala a fin de comprobar la asociación entre variables que se encuentran comprometidas en los procesos de enseñanza-aprendizaje descritos.

\section{Conclusiones}

El presente trabajo tuvo como objetivo la exploración y descripción de dos indicadores de creatividad y su relación con la flexibilidad cognitiva en un grupo de adolescentes en su paso por una institución de innovación educativa, dentro del contexto de educación no formal. El estudio emerge como un intento de dar respuesta a interrogantes surgidos al desempeñar el trabajo cotidiano en la institución educativa.

Los datos obtenidos indicaron que los participantes evaluados presentaron una capacidad creativa alta en comparación con los baremos para adolescentes argentinos. Asimismo, pusieron de manifiesto un alto porcentaje de flexibilidad creativa, la cual a su vez estuvo asociada a la flexibilidad cognitiva. Por lo tanto, el grupo evaluado evidenció un muy buen potencial de creatividad y flexibilidad, aspectos que son por demás interesantes para el desarrollo psicológico en la adolescencia debido a que son factores protectores en situaciones 
de adversidad (Morelato, 2014), además de resultar importantes para la solución de problemas (Corbalán Berná, 2008; Korzeniowski, 2018), la adaptación social y el desarrollo intelectual (Limiñana Gras, 2008).

Sin embargo, las limitaciones que presenta el estudio están vinculadas especialmente a que el mismo se realizó con una muestra no probabilística y pequeña, lo cual no permite generalizar los resultados obtenidos y en consecuencia sólo pueden considerarse preliminares. No obstante, para el alcance propuesto en el proyecto, los resultados son favorables ya que sientan las bases para posteriores investigaciones vinculadas a la temática.

El presente trabajo cuenta con aportes hacia distintas áreas; es de utilidad para el desempeño y funcionamiento de la institución educativa donde se realiza el trabajo ya que, por su condición de novedad, es un dispositivo donde se realizan muchas acciones y muchos cambios, sin dar lugar a una reflexión o evaluación concreta para la educación y el desarrollo. En este sentido, el estudio puede contribuir a hipotetizar acerca de posibles implicancias que estas instituciones tienen en el desarrollo de los niños, niñas y adolescentes a quienes están dirigidas.

Por otra parte, se realiza un aporte en términos de vinculación entre modelos pedagógicos y el desarrollo de los destinatarios. En este punto, aunque existe vasta bibliografía que hace hincapié en las diversas conceptualizaciones acerca de modelos de innovación educativa, sin embargo, son escasas las investigaciones y artículos que puedan describir estas experiencias de modo empírico.

Asimismo, el presente trabajo puede resultar útil para que los actores sociales interesados, o aquellos que desempeñan su trabajo en vinculación con la educación, la niñez y/o la adolescencia, puedan reflexionar en torno a la modalidad de las prácticas, la disposición de los ambientes de trabajo, la planificación y el modo de desempeñar los diversos roles ocupacionales, los cuales pueden favorecer u obstaculizar el desarrollo de esos NNyA y, por tanto, influyen en la construcción de la sociedad.

\section{Referencias}

Corbalán, F.J., Martínez, F., Donolo, D., Tejerina, M., Limiñana, R.M. (2003). CREA Inteligencia Creativa. Una medida cognitiva de la creatividad. Madrid: TEA Ediciones.

Corbalán Berná, J. (2008). ¿De qué se habla cuando hablamos de creatividad? Cuadernos de la Facultad de Humanidades y Ciencias Sociales. Universidad Nacional de Jujuy, (35), 11-21.
Diamond, A. (2014). Want to optimize executive functions and academic outcomes? Simple, just nourish the human spirit. In P. D. Zelazo \& M. D. Sera (Eds.), Minnesota symposia on child psychology: Vol. 37. Developing cognitive control processes: Mechanisms, implications, and interventions ( $\mathrm{p}$. 205-230). John Wiley \& Sons Inc.

Donadel, F. (2019) Innovación educativa y sus implicancias en el desarrollo 
infantojuvenil. [Tesis de licenciatura no publicada]. Universidad del Aconcagua.

Duarte Briceño, E. (diciembre, 2003). Creatividad como un recurso psicológico para niños con necesidades educativas especiales. Sapiens. Revista Universitaria de Investigación, 4(2), 13-32.

Guilford, J. (1950). Creativity. American Psychologist, 5, 444-454.

Hernández Sampieri, R., Baptista Lucio, P y Fernandez Collado, C. (2014) Metodología de la Investigación. México D.F.: Mc. Graw Hill.

Hoyos De Los Ríos, O. L.; Olmos Solís, K. y De Los Reyes Aragón, C. J. (2013). Flexibilidad cognitiva y control inhibitorio: un acercamiento clínico a la comprensión del maltrato entre iguales por abuso de poder. Revista argentina de clínica psicológica, 22(3), 219-228.

IBM Corp. Released 2017. IBM SPSS Statistics for Windows, Version 22.0. Armonk, NY: IBM Corp.

Klimenko, O., Osorio Cadavid, L., Bravo Jaramillo, L., Ocampo Agudelo, S. (2015). Fomento de la capacidad creativa en algunas instituciones educativas de la ciudad de Medellín, Colombia. Revista Psicoespacios, 9 (14) 197-232.

Korzeniowski, C. G. (2011). Desarrollo evolutivo del funcionamiento ejecutivo y su relación con el aprendizaje escolar. [en línea], Revista de Psicología, 7(13). Disponible en: http:// bibliotecadigital.uca.edu.ar/ repositorio/revistas/ desarrollo- evolutivo-funcionamientoejecutivo.pdf

Korzeniowski, C. G. (2018). Las funciones ejecutivas en el estudiante: su comprensión e implementación desde el salón de clases. Informe de la Dirección General de Escuelas, Mendoza, Argentina. (Res ${ }^{\circ} 56$ CGES-18)

León, O. y Montero, I. (2015) Métodos de investigación en Psicología y Educación. Las tradiciones cuantitativa y cualitativa. Madrid: Mc. Graw Hill.

Ley de Educación Nacional $\mathrm{N}^{\circ}$ 26.206. Boletín Oficial de la República Argentina, Buenos Aires, Argentina, 27 de diciembre de 2006.

Liminaña Gras, R. M. (2008). Cuando crear es algo más que un juego: creatividad, fantasía e imaginación en los jóvenes. Cuadernos de la Facultad de Humanidades y Ciencias Sociales. Universidad Nacional de Jujuy. 35, 39-43.

López Martínez, O. (2008). Enseñar creatividad. El espacio educativo. Cuadernos de la Facultad de Humanidades y Ciencias Sociales - Universidad Nacional de Jujuy, (35).

Maddio, S. y Greco, C. (2010). Flexibilidad Cognitiva para Resolver Problemas entre Pares ¿Difiere esta Capacidad en Escolares de Contextos Urbanos y Urbanomarginales? Interamerican Journal of Psychology, 44,(1).

Martí, J., Heydrich, M, Rojas, M y Hernández., A. (2010) Aprendizaje basado en proyectos. Una experiencia de innovación docente. 
Análisis de la creatividad y la flexibilidad cognitiva en adolescentes en un espacio...

Universidad EAFIT, 201011 - 21.

Matus, O., y Gutierrez A. (2012) Habilidades

Blandas: Una ventaja competitiva en la formación tecnológica. GINT Journal of Industrial NeoTechnologies, 33 - 40.

Morelato, G., Carrada, M., \& Cymlich, S. (2014). Creatividad en la infancia: su relación con el desarrollo social. Revista Iberoamericana De Educación, 64(1), 1-10. https:// doi.org/https://doi.org/10.35362/ rie641341

Morelato, G., Carrada, M., e Ison, M. (2013). Creatividad gráfica y Atención focalizada en niños víctimas de maltrato infantil. Liberabit. Revista de Psicología, 19(1): 81-91.

Morelato, G. (2014). Evaluación de factores de Resiliencia en niños argentinos en contextos de vulnerabilidad familiar. Universitas Psychológica, 13(4), 1473-1488. DOI: http:// dx.doi.org/10.11144/Javeriana UPSY13-4.efrn

Morelato, G., Korzeniowski, C., Greco, C. y Ison, M. (2019). Resiliencia Infantil: Intervención para promover recursos en contextos vulnerables. Revista Costarricense de Psicología, 38(2), 205-223. https://dx.doi.org/10.22544/rcps. v38i02.05

Muñoz, W. (2011). Estrategias de estimulación del pensamiento creativo de los estudiantes en el área de educación para el trabajo en la III etapa de educación básica. Memorias Congreso Iberoamericano de Educación 2021, Buenos Aires: Ministerio de Educación de la Nación Argentina.
Muñoz-Sandoval, A. F., Woodcock, R. W., Mc Grez, K. S., y Mather, N. (2005). Batería III Woodcock -Muñoz. Itsaca, IL: Riverside Publishing.

Organización de las Naciones Unidas [ONU] (2016). Innovación educativa. Recuperado de http://unesdoc.unesco.org/ images/0024/002470/247005s.pdf

Organización Mundial de la Salud. (2019) Desarrollo en la adolescencia. Disponible en: https://www.who. int/maternal_child_adolescent/ topics/adolescence/dev/es/.

Saldarriaga-Zambrano, P. J., Bravo Cedeño, G y Loor Rivadeneira, M. (2016) La teoría constructivista de Jean Piaget y su significación para la pedagogía contemporánea. Dominio de las ciencias., 127 - 137.

Siemens, G. (2004) A learning theory for the digital age [en línea]. Recuperado de http://www.elearnspace.org/ Articles/connectivism.htm

Slachevsky Ch, A.; Pérez, C.; Silva, J.; Orellana, G.; Prenafeta, M. L.; Alegria, P. y Peña, G. (2005). Córtex prefrontal y trastornos del comportamiento: modelos explicativos y métodos de evaluación. Revista chilena de neuro-psiquiatría, 43(2), 109-121.

Zavala-Guirado, M. A., González-Castro, I., \& Vázquez-García, M. A. (2019). Modelo de innovación educativa según las experiencias de docentes y estudiantes universitarios. RIDE Revista Iberoamericana Para La Investigación $Y$ El Desarrollo Educativo, 10(20). https://doi. org/10.23913/ride.v10i20.5 